Kragujevac Journal of Mathematics

Volume 41(2) (2017), Pages 295-312.

\title{
ON BERGMAN TYPE INTEGRAL OPERATORS IN ANALYTIC SPACES IN TUBULAR DOMAINS OVER SYMMETRIC CONES
}

\author{
S. KURILENKO ${ }^{1}$ AND R. SHAMOYAN ${ }^{1}$
}

\begin{abstract}
We provide new results on Bergman type integral operators (or Herz type integral operators) in new Herz type analytic spaces in tubular domains over symmetric cones and in products of such type domains. These results are new even in one dimensional case.
\end{abstract}

\section{IntRoduction AND PRELIMINARIES}

The goal of this paper is to provide new results related with the Bergman type projection in tubular domains over symmetric cones in some new Herz type spaces and to study the action of closely related new integral Herz type operators in some new Herz type spaces of analytic functions in tubular domains over symmetric cones and in more general products of such type tubular domains.

We first add some definitions, known facts for these domains to use them partially in our paper later. We also add some known preliminary facts that needed for the completeness of our exposition.

Let $T_{\Omega}=V+i \Omega$ be the tube domain over an irreducible symmetric cone $\Omega$ in the complexification $V^{\mathbb{C}}$ of an $n$-dimensional Euclidean space $\widetilde{V}$. Following the notation of [13] and [5] we denote the rank of the cone $\Omega$ by $r$ and by $\Delta$ the determinant function on $\widetilde{V}$. Letting $\widetilde{V}=\mathbb{R}^{n}$, we have as an example of a symmetric cone on $\mathbb{R}^{n}$ the forward light cone $\Lambda_{n}$ defined for $n \geq 3$ by

$$
\Lambda_{n}=\left\{y \in \mathbb{R}^{n}: y_{1}^{2}-\cdots-y_{n}^{2}>0, y_{1}>0\right\} .
$$

Key words and phrases. Tubular domains over symmetric cones, Herz type spaces, Bergman type integral operators

2010 Mathematics Subject Classification. Primary: 32A07. Secondary: 32A10, 32A07.

Received: January 29, 2016.

Revised: September 3, 2016. 
Light cones have rank 2. The determinant function in this case is given by the Lorentz form

(see, for example, [5]).

$$
\Delta(y)=y_{1}^{2}-\cdots-y_{n}^{2}
$$

Let us introduce some convenient notations regarding multi-indices.

If $t=\left(t_{1}, \ldots, t_{m}\right)$, then $t^{\star}=\left(t_{m}, \ldots, t_{1}\right)$ and, for $a \in \mathbb{R}, t+a=\left(t_{1}+a, \ldots, t_{m}+a\right)$. Also, if $t, k \in \mathbb{R}^{m}$, then $t<k$ means $t_{j}<k_{j}$ for all $1 \leq j \leq m$.

We are going to use the following multi-index

$$
g_{0}=\left((j-1) \frac{d}{2}\right)_{1 \leq j \leq r}, \quad \text { where }(r-1) \frac{d}{2}=\frac{n}{r}-1 .
$$

$\mathcal{H}\left(T_{\Omega}\right)$ denotes the space of all holomorphic functions on $T_{\Omega}$. We denote $m$ Cartesian products of tubes by $T_{\Omega}^{m}, T_{\Omega}^{m}=T_{\Omega} \times \cdots \times T_{\Omega}$ the space of all analytic function on this new product domain which are analytic by each variable separately will be denoted by $\mathcal{H}\left(T_{\Omega}^{m}\right)$. In this paper we will be interested on properties of certain analytic subspaces of $\mathcal{H}\left(T_{\Omega}^{m}\right)$. By $m$ here and everywhere below we denote below a natural number bigger than 1. For $\tau \in \mathbb{R}_{+}$and the associated determinant function $\Delta(x)$ we set

$$
\tilde{A}_{\tau}^{\infty}\left(T_{\Omega}\right)=\left\{F \in \mathcal{H}\left(T_{\Omega}\right):\|F\|_{A_{\tau}^{\infty}}=\sup _{x+i y \in T_{\Omega}}|F(x+i y)| \Delta^{\tau}(y)<\infty\right\},
$$

(see [5] and references there). It can be checked that this is a Banach space.

For $1 \leq p, q<+\infty$ and $\nu \in \mathbb{R}$, and $\nu>\frac{n}{r}-1$ we denote by $\tilde{A}_{\nu}^{p, q}\left(T_{\Omega}\right)$ the mixed-norm weighted Bergman space consisting of analytic functions $f$ in $T_{\Omega}$ that

$$
\|F\|_{\tilde{A}_{\nu}^{p, q}}=\left(\int_{\Omega}\left(\int_{\widetilde{V}}|F(x+i y)|^{p} d x\right)^{q / p} \Delta^{\nu}(y) \frac{d y}{\Delta(y)^{n / r}}\right)^{1 / q}<\infty .
$$

This is a Banach space.

Replacing above simply $\tilde{A}$ by $\tilde{L}$ we will get as usual the corresponding larger space of all measurable functions in tube over symmetric cone with the same quazinorm (see $[13,19,20])$. It is known the $\tilde{A}_{\nu}^{p, q}\left(T_{\Omega}\right)$ space is nontrivial if and only if $\nu>\frac{n}{r}-1$ (see [5]). And we will assume this everywhere below. When $p=q$ we write (see [5])

$$
\tilde{A}_{\nu}^{p, q}\left(T_{\Omega}\right)=\tilde{A}_{\nu}^{p}\left(T_{\Omega}\right)
$$

This is the classical weighted Bergman space with usual modification when $p=\infty$. We add some notions on Bergman type analytic function spaces on products of tubular domains.

Let $T_{\Omega}^{m}=T_{\Omega} \times \cdots \times T_{\Omega}$. To define related two Bergman-type spaces $\tilde{A}_{\nu}^{p}\left(T_{\Omega}^{m}\right)$ and $\tilde{A}_{\tau}^{\infty}\left(T_{\Omega}^{m}\right)$ ( $\nu$ and $\tau$ can be also vectors) in m-products of tube domains $T_{\Omega}^{m}$ we follow standard procedure which is well-known in case of unit disk and unit ball see for example [11]. Namely we consider analytic $F$ functions $F=F\left(z_{1}, \ldots z_{m}\right)$ which are analytic by each $z_{j}, j=1, \ldots, m$ variable, and where each such variable belongs to $T_{\Omega}$ 
tube and define as $H\left(T_{\Omega}^{m}\right)$ the space of all such functions. For example we set, for all $z_{j}=x_{j}+y_{j}, \tau_{j} \in R, j=1, \ldots, m, F(z)=F\left(z_{1}, \ldots, z_{m}\right), \tau=\left(\tau_{1}, \ldots, \tau_{m}\right)$

$$
\tilde{A}_{\tau}^{\infty}\left(T_{\Omega}^{m}\right)=\left\{F \in \mathcal{H}\left(T_{\Omega}^{m}\right):\|F\|_{\tilde{A}_{\tau}^{\infty}}=\sup _{x+i y \in T_{\Omega}^{m}}|F(x+i y)| \Delta^{\tau}(y)<\infty\right\},
$$

where

$$
|F(x+i y)|=\left|F\left(x_{1}+i y_{1}, \ldots, x_{m}+i y_{m}\right)\right|,
$$

and $\Delta^{\tau}(y)$ is a product of $\mathrm{m}$ one-dimensional $\Delta^{\tau_{j}}\left(y_{j}\right)$ functions, $j=1, \ldots, m$. Similarly the Bergman space $\tilde{A}_{\tau}^{p}$ can be defined on products of tubes for all $\tau=\left(\tau_{1}, \ldots, \tau_{m}\right), \tau_{j}>$ $\frac{n}{r}-1, j=1, \ldots, m$. It can be shown that all spaces are Banach spaces. Replacing above simply $\tilde{A}$ by $\tilde{L}$ we will get as usual the corresponding larger space of all measurable functions in products of tubes over symmetric cone with the same quazinorm.

The (weighted) Bergman projection $P_{\nu}$ is the orthogonal projection from the Hilbert space $\tilde{L}_{\nu}^{2}\left(T_{\Omega}\right)$ onto its closed subspace $\tilde{A}_{\nu}^{2}\left(T_{\Omega}\right)$ and it is given by the following integral formula (see [5])

$$
P_{\nu} f(z)=C_{\nu} \int_{T_{\Omega}} B_{\nu}(z, w) f(w) d \tilde{V}_{\nu}(w)
$$

where

$$
B_{\nu}(z, w)=C_{\nu} \Delta^{-\left(\nu+\frac{n}{r}\right)}((z-\bar{w}) / i)
$$

is the Bergman reproducing kernel for

$$
\tilde{A}_{\nu}^{2}\left(T_{\Omega}\right)
$$

$($ see $[5,13])$.

Here we used the notation

$$
d \tilde{V}_{\nu}(w)=\Delta^{\nu-\frac{n}{r}}(v) d u d v
$$

Below and here we use constantly the following notations $w=u+i v \in T_{\Omega}$ and also $z=x+i y \in T_{\Omega}$.

Hence for any analytic function from $\tilde{A}_{\nu}^{2}\left(T_{\Omega}\right)$ the following reproducing integral formula is valid (see also [5])

$$
f(z)=C_{\nu} \int_{T_{\Omega}} B_{\nu}(z, w) f(w) d \tilde{V}_{\nu}(w) .
$$

Note these assertions have direct copies in simpler cases of analytic function spaces in unit disk, polydisk, unit ball, upper half-space $C_{+}$and in spaces of harmonic functions in the unit ball or upper half-space of the Euclidean space $R^{n}$. These classical facts are well-known and can be found, for example, in [11] and in some items from references there.

Let us first recall the following known basic integrability properties for the determinant function, which appeared already above in definitions. Below we denote by $\Delta_{s}$ the generalized power function (see for this function $[5,13]$ ). 


\section{Lemma 1.1.}

1) The integral

$$
J_{\alpha}(y)=\int_{\mathbb{R}^{n}}\left|\Delta^{-\alpha}\left(\frac{x+i y}{i}\right)\right| d x
$$

converges if and only if $\alpha>2 \frac{n}{r}-1$. In that case

$$
J_{\alpha}(y)=\widetilde{C}_{\alpha} \Delta^{-\alpha+n / r}(y), \quad \alpha \in R, y \in \Omega .
$$

2) Let $\alpha \in \mathbb{C}^{r}$ and $y \in \Omega$. For any multi-indices $s$ and $\beta$ and $t \in \Omega$ the function

$$
y \mapsto \Delta_{\beta}(y+t) \Delta_{s}(y)
$$

belongs to $L^{1}\left(\Omega, \frac{d y}{\Delta^{n / r}(y)}\right)$ if and only if $\operatorname{Re} s>g_{0}$ and $\operatorname{Re}(s+\beta)<-g_{0}^{*}$. In that case we have

$$
\int_{\Omega} \Delta_{\beta}(y+t) \Delta_{s}(y) \frac{d y}{\Delta^{n / r}(y)}=\widetilde{C}_{\beta, s} \Delta_{s+\beta}(t) .
$$

We refer to Corollary 2.18 and Corollary 2.19 of [10] for the proof of the above lemma or [5]. As a corollary of one dimensional version of second estimate and first estimate (see, for example, [20], [19, Theorem 3.9]) we obtain the following vital Forelli-Rudin type estimate for Bergman kernel (1.2) which we will use in proof of all our main results [19].

\section{Lemma 1.2.}

$$
\int_{T_{\Omega}} \Delta^{\beta}(y)\left|B_{\alpha+\beta+\frac{n}{r}}(z, w)\right| d V(z) \leq C \Delta^{-\alpha}(v),
$$

where $\beta>-1, \alpha>\frac{n}{r}-1, z=x+i y$, and $w=u+i v$ (see [20]).

We note also that see [20]

$$
|f(x+i y)| \Delta^{\frac{n}{r p}+\frac{\nu}{q}}(y) \leq c_{p, q, r, \nu}\|f\|_{\tilde{A}_{\nu}^{p, q},} \quad 1 \leq p, q<\infty, \nu>\frac{n}{r}-1 .
$$

Finally for completeness we provide very vital Whitney decomposition of tubular domain over symmetric cones based on Bergman balls (see [5,6]). It was used during many proofs of various assertions (see for example $[5,6]$ ).

Lemma $1.3([4,5])$. Given $\delta \in(0 ; 1)$ there exists a sequence of points $\left\{z_{j}\right\}$ in $T_{\Omega}$ called $\delta$-lattice such that calling $\left\{B_{j}\right\}$ and $\left\{B_{j}^{\prime}\right\}$ the Bergman balls with center $z_{j}$ and radius $\delta$ and $\delta / 2$ respectively then

A) the balls $\left\{B_{j}^{\prime}\right\}$ are pairwise disjoint;

B) the balls $\left\{B_{j}\right\}$ cover $T_{\Omega}$ with finite overlapping;

C) $\int_{B_{j}\left(z_{j}, \delta\right)} \Delta^{s}(y) d V(z) \asymp \int_{B_{j}^{\prime}\left(z_{j}, \delta\right)} \Delta^{s}(y) d V(z)=\widetilde{C}_{\delta} \Delta^{2 \frac{n}{r}+s}\left(\operatorname{Im} z_{j}\right)$;

where $s>\frac{n}{r}-1, J=\left|B_{\delta}\left(z_{j}\right)\right| \asymp \Delta^{\frac{2 n}{r}}\left(\operatorname{Im} z_{j}\right), j=1, \ldots, m, J \asymp \Delta^{\frac{2 n}{r}}(\operatorname{Im} w)$, and $w \in B_{\delta}\left(z_{j}\right)$. 
Let $d v\left(z_{1}, \ldots, z_{m}\right)=\prod_{j=1}^{m} d v\left(z_{j}\right)=d v(\vec{z}), z_{j} \in T_{\Omega}, j=1, \ldots, m$ be the normalized Lebeques measure on product domain. We denote as usual by $d v_{\gamma}=\delta^{\gamma}(z) d v(z)$ the weighted Lebegues measure on $T_{\Omega}$ domain and similarly on products of such domains using products of $\Delta$ functions in a standard way for all $\gamma>-1$. Much work was done in recent decades in the theory of analytic function spaces in tubular domains over symmetric cones. Various interesting results were provided related with Bergman type integral operators on such type spaces. We refer to [5] and [20] and various references there only for a short portion of such type results.

In this section we define new scales of Herz type analytic function spaces on more general products of such tubes and based on that we study the action of some new Herz type integral operators defined on more general products of tubular domains over symmetric cones. Note we always assume the symbols of these integral operators belong as usual formally to $L^{1}$ class in tubular domains $T_{\Omega}$ or to same spaces on products of such tubular domains $\left(T_{\Omega}\right)^{m}$. To be more precise we define new analytic Herz-type spaces on products of tubular domains in $\mathbb{C}^{n}$ via Bergman balls (or products of such balls) in tube following the same ideas we used in our previous work related with Herz type spaces and operators and Bergman type integral operators in context of bounded strongly pseudoconvex domains with smooth boundary and products of such domains (note here using standard sign of vector on lower indexes everywhere below we get as usual a little bit more general versions of these analytic spaces-and this is a standard extension in literature). We refer to [23] for these results in bounded strongly pseudoconvex domains related with Trace problem. We refer to $[1-3,7,8,12,15-18,24]$ for various recent nice results related with the Bergman and other integral operators in various domains in analytic function spaces in higher dimension. We provide now several recently obtained results in this area on Bergman type projections in tube. The weighted Bergman projection $P_{\nu}$ is the orthogonal projection from the Hilbert space $\tilde{L}_{\nu}^{2}\left(T_{\Omega}\right)$ onto its closed subspace $\tilde{A}_{\nu}^{2}\left(T_{\Omega}\right)$ and it is given by the integral formula

$$
\left(P_{\nu} f\right)(z)=\int_{T_{\Omega}} B_{\nu}(z, w) f(w) \Delta^{\nu-\frac{n}{r}}(\operatorname{Im} w) d v(w)
$$

$z \in T_{\Omega}$ and $\nu>\frac{n}{r}-1$ (see $[4-6,19,20]$ ).

The $\tilde{L}_{\nu}^{p, q}$ boundedness of the Bergman projection $P_{\nu}$ is still an open problem and has attracted a lot of attention in recent years. Today it is only known that this projection extends to a bounded operator on $\tilde{L}_{\nu}^{p, q}$ for general symmetric cones for the range $1 \leq p<\infty, q_{\nu, p}^{\prime}<q<q_{\nu, p} ; q_{\nu, p}=\min \left\{p, p^{\prime}\right\} q_{\nu}, q_{\nu}=1+\frac{\nu}{n / r-1}$ and $\frac{1}{p}+\frac{1}{p^{\prime}}=1$ (see $[19,20])$.

The importance can be seen for example from the following fact. If $P_{\nu}$ extends to a bounded operator on $\tilde{L}_{\nu}^{p, q}$ then the topological dual space $\left(\tilde{A}_{\nu}^{p, q}\right)^{*}$ of the Bergman space $\tilde{A}_{\nu}^{p, q}$ identifies with $\tilde{A}_{\nu}^{p^{\prime}, q^{\prime}}$ under the integral pairing

$$
\langle f, g\rangle_{\nu}=\int_{T_{\Omega}} f(z) \overline{g(z)} \Delta^{\nu-\frac{n}{r}}(\operatorname{Im} z) d v(z),
$$


$f \in \tilde{A}_{\nu}^{p, q}, g \in \tilde{A}_{\nu}^{p^{\prime}, q^{\prime}}$ (see $\left.[19,20]\right)$. Let $\beta>-1, \gamma>0$, and $\alpha>0$. Let also

$$
\begin{aligned}
& \left(T_{\alpha, \beta, \gamma} f\right)(z)=\Delta^{\alpha}(\operatorname{Im} z) \int_{T_{\Omega}} B_{\gamma}(z, w) f(w) \Delta^{\beta}(\operatorname{Im} w) d v(w), \\
& \left(T_{\alpha, \beta, \gamma}^{+} f\right)(z)=\Delta^{\alpha}(\operatorname{Im} z) \int_{T_{\Omega}}\left|B_{\gamma}(z, w)\right| f(w) \Delta^{\beta}(\operatorname{Im} w) d v(w),
\end{aligned}
$$

$z \in T_{\Omega}, f \in \tilde{L}^{1}\left(T_{\Omega}\right)$. The following assertions were proved in recently in [19].

Theorem B ([19]). There are $\nu_{1}=\nu_{1}(\alpha, n, r, q) ; \nu_{2}=\nu_{2}(\alpha, n, r, q)$ so that for $1 \leq$ $p, q<\infty, \nu \in R, \gamma=\alpha+\beta+\frac{n}{r}, \alpha+\beta>-1$ the $T_{\alpha, \beta, \gamma}^{+}$operator is a bounded operator on $\tilde{L}_{\nu}^{p, q}\left(T_{\Omega}\right)$ for all $\nu \in\left(\nu_{1}, \nu_{2}\right)$.

Theorem C ([19]). Let $\left(Q^{+}\right)$be $\left(T_{\alpha, \beta, \gamma}^{+}\right)$operator for $\alpha=0, \gamma=\nu+m ; \beta=\nu-\frac{n}{r}$. Then $\left(Q^{+}\right)$for $\nu+m>\frac{n}{r}-1,1 \leq p, q<\infty$, is a bounded operator from $\tilde{L}_{\nu}^{p, q}$ to $\tilde{L}_{\nu+m q}^{p, q}$ if $\nu \in\left(\nu_{1}, \nu_{2}\right)$ for some $\nu_{1}=\nu_{1}(p, q, n, r, \nu) ; \nu_{2}=\nu_{2}(p, q, n, r, \nu)$, and $\left(T_{\alpha, \beta, \gamma}^{+}\right)$is a bounded operator on $L^{\infty}$ if $\alpha>\frac{n}{r}-1, \beta>-1, \gamma=\alpha+\beta+\frac{n}{r}$. The same is valid for $T_{\alpha, \beta, \gamma}$ operator.

By $B(z, R)$ we denote as usual the Bergman ball with center $z$ and radius $R$ in $T_{\Omega}$ (we refer for example to [19] for standard definitions of these balls). We define several new function spaces for $1 \leq p<\infty$ assuming $\nu>-1$, and $\nu_{j}>-1, j=1, \ldots, m$ in tubular domains and in product of tubular domains as follows

$$
\begin{aligned}
& L_{\tau}^{p}\left(T_{\Omega}\right):\left(\int_{T_{\Omega}} \int_{B(\tilde{w}, R)}|f(w)|^{p} \Delta^{\tau}(\operatorname{Im} w) d v(w) d v(\tilde{w})\right)^{\frac{1}{p}}<+\infty, \\
& L_{\tau}^{p}\left(T_{\Omega}^{m}\right): \\
& \left(\int_{T_{\Omega}^{m}} \int_{B\left(\tilde{w}_{1}, R\right)} \ldots \int_{B\left(\tilde{w}_{m}, R\right)}\left|f\left(w_{1}, \ldots, w_{m}\right)\right|^{p} \prod_{j=1}^{m}\left(\Delta^{\tau_{j}}\left(\operatorname{Im} w_{j}\right) d v\left(w_{j}\right)\right) \prod_{j=1}^{m} d v\left(\tilde{w}_{j}\right)\right)^{\frac{1}{p}}<+\infty, \\
& \left(L_{\vec{\nu}}^{p}\left(T_{\Omega}^{m}\right)\right)_{1}: \\
& \left(\int_{T_{\Omega}} \ldots \int_{T_{\Omega}}\left|f\left(w_{1}, \ldots, w_{m}\right)\right|^{p} \Delta^{\nu_{1}}\left(\operatorname{Im} w_{1}\right) \ldots \Delta^{\nu_{m}}\left(\operatorname{Im} w_{m}\right) d v\left(w_{1}\right) \ldots d v\left(w_{m}\right)\right)^{\frac{1}{p}}<+\infty .
\end{aligned}
$$

Finally for $\nu_{j}>0, j=1, \ldots, m, 1 \leq p<\infty, s>0$ we define

$$
\begin{aligned}
\left(L_{\vec{\nu}, \vec{s}}^{p}\left(T_{\Omega}^{m}\right)\right)_{2}: & \left(\int_{T_{\Omega}} \ldots \int_{T_{\Omega}} \int_{B\left(\tilde{z}_{1}, R\right)} \ldots \int_{B\left(\tilde{z}_{m}, R\right)}\left|f\left(z_{1}, \ldots, z_{m}\right)\right|^{p} \prod_{j=1}^{m} \Delta^{\nu_{j}-\frac{n}{r}}\left(z_{j}\right) d v\left(z_{j}\right) \times\right. \\
& \left.\times \prod_{j=1}^{m} \Delta^{s_{j}-\frac{n}{r}}\left(\operatorname{Im} \tilde{z}_{j}\right) d v\left(\tilde{z}_{j}\right)\right)^{\frac{1}{p}}<+\infty .
\end{aligned}
$$

Replacing $L$ by $A$ as usual we get analytic subspaces of these function classes in tube and in products of tubular domains. It is easy to note the forth space for particular 
values of parameters coincide with second space but we will follow this notation. We also define new Herz type integral operator for positive $\alpha_{j}, j=1, \ldots, m$ and for all $\beta>-1$ and $\gamma>-1$,

$$
\left[T_{\vec{\alpha}, \beta, \gamma}(g)\right]\left(z_{1}, \ldots, z_{m}\right)=\int_{T_{\Omega}} \int_{B(\tilde{w}, R)} \frac{g(w)\left[\Delta^{\beta}(\operatorname{Im} w)\right] d v(w)}{\left[\prod_{j=1}^{m} \Delta^{\alpha_{j}}\left(\frac{z_{j}-\bar{w}}{i}\right)\right]} \Delta^{\gamma}(\operatorname{Im} \tilde{w}) d v(\tilde{w}),
$$

where $z_{j} \in T_{\Omega}, j=1, \ldots, m$. We need also a known lemma. It is the base of our proofs and can be seen in [20] and also in [19].

Lemma 1.4. For $\beta>-1, \alpha_{1}>\beta+\frac{2 n}{r}$ we have

$$
\int_{B(\tilde{w}, R)} \frac{\left[\Delta^{\beta}(\operatorname{Im} w)\right] d v(w)}{\left|\Delta^{\alpha_{1}}\left(\frac{w-z}{i}\right)\right|} \leq \frac{c}{\mid \Delta^{\alpha_{1}-\beta-\frac{2 n}{r}\left(\frac{\tilde{w}-z}{i}\right) \mid}},
$$

$z, \tilde{w} \in T_{\Omega}$.

Now for completeness of our exposition we now formulate some assertions related to our results in other domains for Herz-type analytic spaces which were obtained recently namely some complete analogues of our theorems for simpler domains. Let $D$ be unit disk on a complex plane $C$ and $d m_{2}$ be the Lebegues measure on $D$. Let $D^{n}$ be the unit polydisk and $H\left(D^{n}\right)$ be the space of all analytic functions in $D^{n}$, let $T^{n}$ be torus and $d \sigma$ is Lebegues measure on it. For

$$
(G f)(\vec{z})=\int_{D} \frac{f(w)(1-|w|)^{s-1} d m_{2}(w)}{\prod_{j=1}^{n}\left(1-z_{j} \bar{w}\right)^{\frac{s+1}{n}}}, \quad s>s_{0},
$$

for fixed large enough $s_{0}$. It was shown in [14]

$$
\|(G f)(z)\|_{K_{\alpha, \beta}^{p, q}} \leq\|f\|_{H_{\beta+\frac{q}{p}(|\alpha|+2 n-1)}^{p, q}},
$$

where $|\alpha|=\sum_{j=1}^{n} \alpha_{j}, 0<p, q<\infty, \beta>-1, \alpha_{j}>-1, j=1, \ldots, n$. Where

$$
\begin{aligned}
K_{\alpha, \beta}^{p, q}\left(D^{n}\right)= & \left\{f \in H\left(D^{n}\right): \int_{0}^{1}\left(\int_{\left|z_{1}\right|<r} \cdots \int_{\left|z_{n}\right|<r}|f(z)|^{p} \prod_{j=1}^{n}\left(1-\left|z_{j}\right|\right)^{\alpha_{j}} d m_{n}(z)\right)^{\frac{q}{p}}\right. \\
& \left.\times(1-r)^{\beta} d r<\infty\right\},
\end{aligned}
$$

$0<p, q<\infty, \beta>-1, \alpha_{j}>-1$, and $j=1, \ldots, n$ is a Herz type space of analytic functions in the unit polydisk

$$
\begin{aligned}
H_{\alpha}^{p, q}\left(D^{n}\right)= & \left\{f \in H\left(D^{n}\right): \int_{0}^{1} \cdots \int_{0}^{1}\left(\prod_{j=1}^{n}\left(1-r_{j}^{2}\right)^{\alpha_{j}}\right)\left(\int_{T^{n}}|f(r \zeta)|^{p} d \sigma(\zeta)\right)^{\frac{q}{p}}\right. \\
& \left.\times d r_{1} \ldots d r_{n}<\infty\right\}
\end{aligned}
$$


where $0<p, q<\infty, \beta>-1, \alpha_{j}>-1, j=1, \ldots, n$.

This type result later were found and proved also even in context of more general domain namely the unit ball $B=\{z:|z|<1\}$ in paper [21] and in [23] in context of bounded strongly pseudoconvex domains with smooth boundary.

We now discuss another similar result on the action of Bergman type integral operator in analytic Herz type spaces in the unit ball. Note first for measurable function $f$ in the unit ball we have

$$
\int_{B}|f|(1-|z|)^{\alpha} d \mu(z) \asymp \int_{S} \int_{\Gamma_{t}(\zeta)}|f|(1-|z|)^{\alpha-n} d \mu(z) d \zeta,
$$

where $d \mu$ is a positive Borel measure on $B, \Gamma_{t}(\zeta)=\{z \in B:|1-\bar{\zeta} z|<t(1-|z|)\}$, $t>1, \zeta \in S, S=\{|z|=1\}$, where $\alpha>-1$.

Using that we have the following estimate for Bergman type integral operators (see

$$
\begin{aligned}
& \int_{S} \int_{\Gamma_{t}(\zeta)} \cdots \int_{S} \int_{\Gamma_{t}(\zeta)}\left|\left(T_{a, b} f\right)\left(z_{1}, \ldots, z_{m}\right)\right|^{p} \prod_{j=1}^{m}\left(1-\left|z_{j}\right|\right)^{s_{j}-n} d v\left(z_{1}\right) \ldots d v\left(z_{m}\right) d \sigma(\vec{\zeta}) \\
\leq & c \int_{S} \int_{\Gamma_{t}(\zeta)}|f(w)|^{p}\left(1-|w|^{2}\right)^{(m-1)(n+1)+\sum_{j=1}^{m} s_{j}-n} d v(w) d \sigma(\zeta),
\end{aligned}
$$

where $1 \leq p<\infty, s_{j}>-1, j=1, \ldots, m, b_{j}>b_{0}, a_{j}>a_{0}, j=1, \ldots, m, f \in$ $L^{1}(B, d \nu)$, and

$$
\left(T_{a, b} f\right)\left(z_{1}, \ldots, z_{m}\right)=\prod_{j=1}^{m}\left(1-\left|z_{j}\right|^{2}\right)^{a_{j}} \int_{S} \int_{\Gamma_{t}(\zeta)} \frac{f(w)\left(1-|w|^{2}\right)^{-2 n-1+\sum_{j=1}^{m} b_{j}} d v(w) d \zeta}{\prod_{j=1}^{m}\left|1-\left\langle z_{j}, w\right\rangle\right|^{a_{j}+b_{j}}} .
$$

The natural question is the following. Let $X_{\xi} \subset B, \zeta \in S$ be a set then can we find other estimates like (1.4) of the following type

$$
\begin{aligned}
& \int_{S} \int_{S} \int_{X_{\zeta}} \ldots \int_{X_{\zeta}}\left|\left(T_{a, b} f\right)\left(z_{1}, \ldots, z_{m}\right)\right|^{p} d \mu_{1}\left(z_{1}\right) \ldots d \mu_{m}\left(z_{m}\right) d \sigma(\zeta) \\
\leq & c \int_{S} \int_{X_{\zeta}}|f(w)|^{p} d \mu(w) d \zeta
\end{aligned}
$$

for some functions $\mu_{1}\left(z_{1}\right), \ldots, \mu_{m}\left(z_{m}\right)$ and

$$
\left(T_{a, b} f\right)\left(z_{1}, \ldots, z_{m}\right)=g(z) \int_{S} \int_{X_{\zeta}}(f(w)) \prod_{j=1}^{m} \tilde{\mu}_{j}\left(z_{j}, w\right) d \mu(w)
$$

where $g(\vec{z}), \tilde{\mu}_{j}\left(z_{j}, w\right), \mu(w)$ are certain fixed measures and (or) functions.

The same problem can be posed for any set $Y_{z} \subset B\{|z|<1\}, z \in B$. And then integration move above via $\int_{B} \int_{Y_{z}}$ instead of $\int_{S} \int_{X_{\xi}}$ (these last spaces are Herz type function spaces). 
Moreover questions of this type can be posed in other domains in $C^{n}$ also (tube, pseudoconvex domains etc.).

In this paper we provide some solutions of such type problems in tubular domains over symmetric cones where we consider spaces and operators of new Herz-type defined with the help of Bergman metric ball in tube over symmetric cone $T_{\Omega}$. These type results can be applied for example to trace problems and also to other problems in function theory and may have also other interesting applications.

Here is a complete analogue of our results in the unit ball (typical bounded Siegel domain of second type). We refer the reader to [11] for basics on function theory in the unit ball.

Let $H(B)$ be the space of all analytic functions in the unit ball and let $D(z, r)$ be the Bergman ball in $B$ in Bergman metric (see, for example, [11] and references there). We denote the product of balls by $B^{m}, m \in N$, and the related space of all analytic function in it by $H\left(B^{m}\right)$. Let $A_{\alpha}^{P}$ be the usual Bergman class in the unit ball (see for example [21]). Let further

$$
\begin{aligned}
K_{\alpha, \beta}^{p, q}\left(B^{m}\right)= & \left\{f \in H(B \times \cdots \times B): \int_{B} \ldots \int_{B}\left(\int_{D\left(z_{1}, r\right)} \cdots \int_{D\left(z_{1}, r\right)}\left|f\left(\tilde{z}_{1}, \ldots, \tilde{z}_{m}\right)\right|^{p} \times\right.\right. \\
& \left.\times\left(1-\left|\tilde{z}_{1}\right|\right)^{\alpha_{1}} \ldots\left(1-\left|\tilde{z}_{m}\right|\right)^{\alpha_{m}} d v\left(\tilde{z}_{1}\right) \ldots d v\left(\tilde{z}_{m}\right)\right) \\
& \left.\times\left(1-\left|z_{1}\right|\right)^{\beta_{1}} \ldots\left(1-\left|z_{m}\right|\right)^{\beta_{m}} d v\left(z_{1}\right) \ldots d v\left(z_{m}\right)<\infty\right\},
\end{aligned}
$$

where $p \in(0, \infty), \alpha_{j}>-1, \beta_{j}>-1$, and $j=1, \ldots, m$. Then $\|\tilde{T} f\|_{K_{t, \beta}^{p, p}} \leq c\|f\|_{A_{\alpha}^{p}}$, $0<p<\infty, \alpha>-1, \beta_{j}>-1, j=1, \ldots, m, t_{j}>-1, j=1, \ldots, m, n \in \mathbb{N}$, $0<p<\infty, \alpha=\sum_{j=1}^{m}\left(\beta_{j}+2(n+1)+t_{j}\right)-(n+1)$, where

$$
(\tilde{T} f)=F\left(z_{1}, \ldots, z_{m}\right)=c\left(\int_{B} \frac{f(w)(1-|w|)^{s} d v(w)}{\prod_{j=1}^{m}\left(1-\left\langle\bar{w}, z_{j}\right\rangle\right)^{\frac{s+1+n}{m}}}\right), \quad z_{j} \in B,
$$

and $j=1, \ldots, n$.

\section{Bergman-Type Integral Operators and Herz Type Spaces in Tubular Domains Over Symmetric Cones}

In this section we formulate our main results. In the next four theorems we provide some information on the action of $T_{\vec{\alpha}, \beta, \gamma}$ Bergman type integral operators in $\left(A_{\vec{\nu}}^{p}\left(T_{\Omega}^{m}\right)\right)_{1}$ and $\left(A_{\vec{\nu}, \vec{s}}^{p}\left(T_{\Omega}^{m}\right)\right)_{2}$ analytic function spaces. The following theorem is valid.

Theorem 2.1. For $1 \leq p<\infty$, the following estimate is valid

$$
\left\|T_{\vec{\alpha}, \beta, \gamma}(g)\right\|_{\left(L_{\vec{\nu}}^{p}\right)_{1}\left(T_{\Omega}^{m}\right)} \leq c\|g\|_{\left(L_{\tau}^{p}\right)\left(T_{\Omega}\right)},
$$

where $\frac{1}{p}+\frac{1}{p^{\prime}}=1, \beta>-1, p^{\prime} \gamma>-1$, and

$$
\alpha_{j}>\frac{\beta}{m}+\frac{2 n}{r m}+\frac{3 n}{r p}-\frac{1}{p}+\frac{3 n}{r p^{\prime} m}-\frac{1}{p^{\prime} m}+\frac{\gamma}{m}, \quad j=1, \ldots, m,
$$


and

$$
\tau=p \beta+\frac{2 n m}{r}-p \sum_{j=1}^{m}\left(\alpha_{j}-\frac{\nu_{j}}{p}\right)+p \gamma+\frac{4 n p}{r p^{\prime}}-\frac{n m}{r p} .
$$

Proof. We need to prove that

$$
\begin{aligned}
J= & \int_{T_{\Omega}} \ldots \int_{T_{\Omega}}\left|\int_{T_{\Omega}} \int_{B(\tilde{w}, R)} \frac{g(w)\left[\Delta^{\beta}(\operatorname{Im} w)\right] d v(w)}{\left[\prod_{j=1}^{m} \Delta^{\alpha_{j}}\left(\frac{z_{j}-\bar{w}}{i}\right)\right]} \Delta^{\gamma}(\operatorname{Im} \tilde{w}) d v(\tilde{w})\right|^{p} \\
& \times \prod_{j=1}^{m} \Delta^{\nu_{j}-\frac{n}{r}}\left(z_{j}\right) d v\left(z_{j}\right) \leq c\|g\|_{\left(L_{\tau}^{p}\right)\left(T_{\Omega}\right)} .
\end{aligned}
$$

Using Holder's inequality we have

$$
\begin{aligned}
\left|T_{\vec{\alpha}, \beta, \gamma}(g)\left(z_{1}, \ldots, z_{m}\right)\right|= & \int_{T_{\Omega}}\left(\int_{B(\tilde{w}, R)} \frac{|g(w)|^{p}\left[\Delta^{\beta}(\operatorname{Im} w)\right] d v(w)}{\left.\mid \prod_{j=1}^{m} \Delta^{p h_{j}\left(\frac{z_{j}-\bar{w}}{i}\right) \mid}\right)^{\frac{1}{p}}}\right. \\
& \times\left(\int_{B(\tilde{w}, R)} \frac{\left[\Delta^{\beta}(\operatorname{Im} w)\right] d v(w)}{\left.\mid \prod_{j=1}^{m} \Delta^{p^{\prime} b_{j}\left(\frac{z_{j}-\bar{w}}{i}\right) \mid}\right)^{\frac{1}{p^{\prime}}} \Delta^{\gamma}(\operatorname{Im} \tilde{w}) d v(\tilde{w}),}\right.
\end{aligned}
$$

where $h_{j}+b_{j}=\alpha_{j}, \frac{1}{p}+\frac{1}{p^{\prime}}=1, j=1, \ldots, m, z_{j} \in T_{\Omega}, j=1, \ldots, m$.

By Lemma 1.4 and Holder's inequality

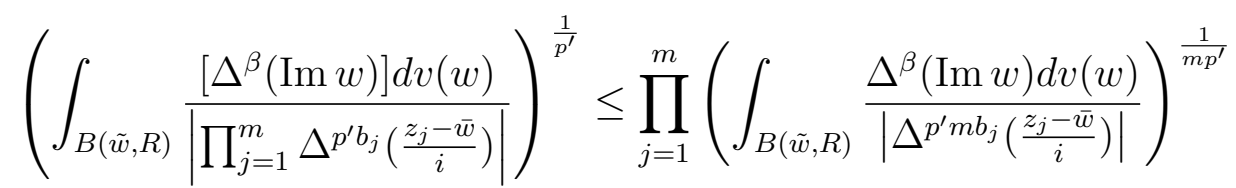

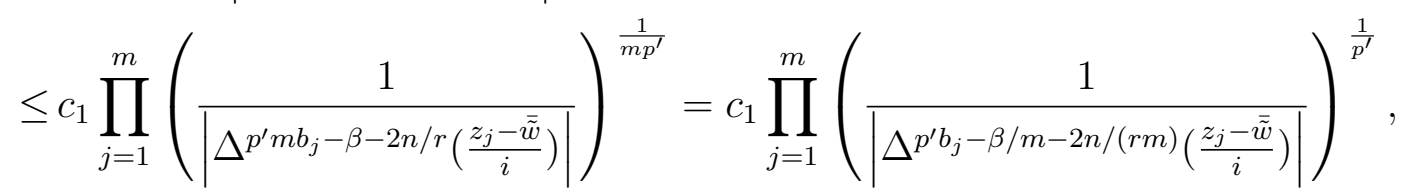

where $\beta>-1$ and $p^{\prime} b_{j}>\frac{\beta}{m}+\frac{2 n}{r m}$. So

$$
\begin{aligned}
\left|T_{\vec{\alpha}, \beta, \gamma}(g)\left(z_{1}, \ldots, z_{m}\right)\right| \leq & c_{1} \int_{T_{\Omega}}\left(\int_{B(\tilde{w}, R)} \frac{|g(w)|^{p}\left[\Delta^{\beta}(\operatorname{Im} w)\right] d v(w)}{\left|\prod_{j=1}^{m} \Delta^{p h}\left(\frac{z_{j}-\bar{w}}{i}\right)\right|}\right)^{\frac{1}{p}} \\
& \times\left(\frac{\Delta^{p^{\prime} \gamma}(\operatorname{Im} \tilde{w})}{\left|\prod_{j=1}^{m} \Delta^{p^{\prime} b_{j}-\beta / m-2 n /(r m)}\left(\frac{z_{j}-\tilde{\tilde{w}}}{i}\right)\right|}\right)^{\frac{1}{p^{\prime}}} d v(\tilde{w})
\end{aligned}
$$

where $z_{j} \in T_{\Omega}, j=1, \ldots, m$. 
Let us use Holder inequality once more

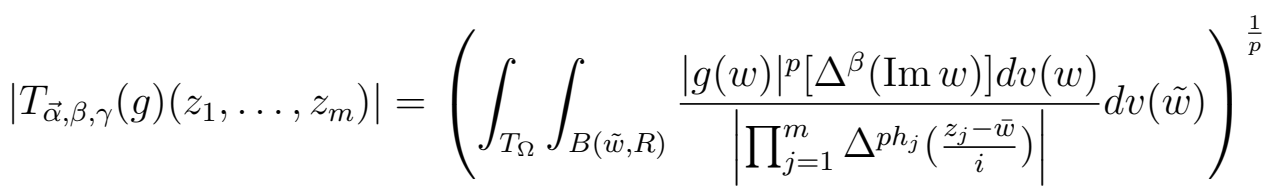

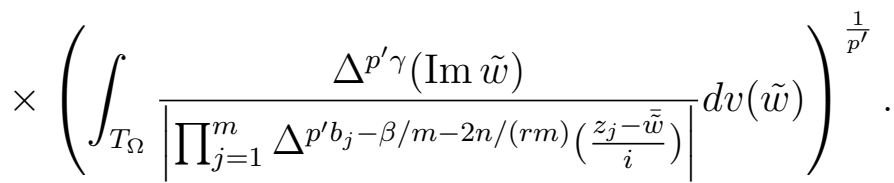

And so

$$
\begin{aligned}
& \left|T_{\vec{\alpha}, \beta, \gamma}(g)\left(z_{1}, \ldots, z_{m}\right)\right|^{p} \leq c_{1} \int_{T_{\Omega}} \int_{B(\tilde{w}, R)} \frac{|g(w)|^{p}\left[\Delta^{\beta}(\operatorname{Im} w)\right] d v(w)}{\left|\prod_{j=1}^{m} \Delta^{p h_{j}}\left(\frac{z_{j}-\bar{w}}{i}\right)\right|} d v(\tilde{w})
\end{aligned}
$$

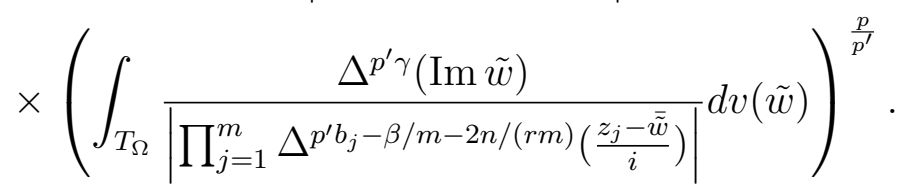

But with respect to (1.2) and Holder's inequality

$$
\begin{aligned}
& \left(\int_{T_{\Omega}} \frac{\Delta^{p^{\prime} \gamma}(\operatorname{Im} \tilde{w})}{\left|\prod_{j=1}^{m} \Delta^{p^{\prime} b_{j}-\beta / m-2 n /(r m)}\left(\frac{z_{j}-\tilde{\tilde{w}}}{i}\right)\right|} d v(\tilde{w})\right)^{\frac{p}{p^{\prime}}} \\
\leq & \prod_{j=1}^{m}\left(\int_{T_{\Omega}} \frac{\Delta^{p^{\prime} \gamma}(\operatorname{Im} \tilde{w})}{\left|\Delta^{p^{\prime} m b_{j}-\beta-2 n / r}\left(\frac{z_{j}-\tilde{\tilde{w}}}{i}\right)\right|} d v(\tilde{w})\right)^{\frac{p}{m p^{\prime}}} \\
\leq & c_{2} \prod_{j=1}^{m} \Delta^{\left(p^{\prime} \gamma-p^{\prime} m b_{j}+\beta+4 n / r\right) p /\left(m p^{\prime}\right)}\left(\operatorname{Im} z_{j}\right) \\
= & c_{2} \prod_{j=1}^{m} \Delta^{\left(p \gamma / m-p b_{j}+\beta p /\left(m p^{\prime}\right)+4 n p /\left(r m p^{\prime}\right)\right.}\left(\operatorname{Im} z_{j}\right) .
\end{aligned}
$$

Where $p^{\prime} \gamma>-1$ and

$$
p^{\prime} m b_{j}-p^{\prime} \gamma-\beta-\frac{4 n}{r}>\frac{n}{r}-1 .
$$

Denote now $p \gamma / m-p b_{j}+\beta p /\left(m p^{\prime}\right)+4 n p /\left(r m p^{\prime}\right)=-\nu_{j}+\frac{n}{r}, j=1, \ldots, m$. Then we have that

$$
b_{j}=\frac{\nu_{j}}{p}+\frac{\gamma}{m}+\frac{\beta}{m p^{\prime}}+\frac{4 n}{r m p^{\prime}}-\frac{n}{r p} .
$$

In this case we have

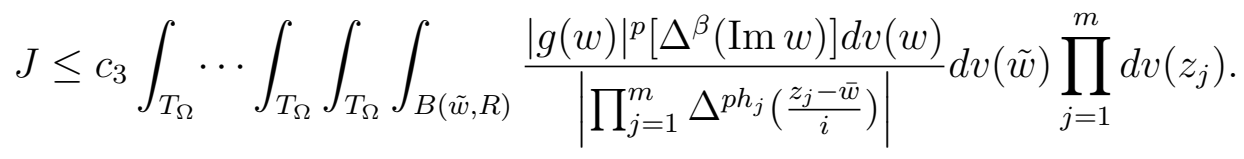


Using Fubbini's theorem and we obtain

$$
\begin{aligned}
J & \leq c_{3} \int_{T_{\Omega}} \int_{B(\tilde{w}, R)}|g(w)|^{p} \prod_{j=1}^{m} \int_{T_{\Omega}} \frac{d v\left(z_{j}\right)}{\mid \Delta^{p h_{j}\left(\frac{z_{j}-\bar{w}}{i}\right) \mid} \Delta^{\beta}(\operatorname{Im} w) d v(w) d v(\tilde{w})} \\
& \leq c_{4} \int_{T_{\Omega}} \int_{B(\tilde{w}, R) \mid}|g(w)|^{p} \Delta^{\beta+2 n m / r-p \sum_{j=1}^{m} h_{j}}(\operatorname{Im} w) d v(w) d v(\tilde{w}) \\
& =c_{4} \int_{T_{\Omega}} \int_{B(\tilde{w}, R) \mid}|g(w)|^{p} \Delta^{\tau}(\operatorname{Im} w) d v(w) d v(w) d v(\tilde{w}),
\end{aligned}
$$

where $\tau=\beta-p \sum_{j=1}^{m} h_{j}+2 n m / r$ and

$$
p h_{j}-\frac{\beta}{m}-\frac{2 n}{r m}-\frac{2 n}{r}>\frac{n}{r-1} .
$$

Using (2.2) and fact that $h_{j}=\alpha_{j}-b_{j}$ we have

$$
\tau=\beta+\frac{2 n m}{r}-p \sum_{j=1}^{m}\left(\alpha_{j}-\frac{\nu_{j}}{p}\right)+p \gamma+\frac{p \beta}{p^{\prime}}+\frac{4 n p}{r p^{\prime}}-\frac{n m}{r p} .
$$

Moreover from (2.1) and (2.3) we have some restriction on $\alpha_{j}$ :

$$
\alpha_{j}>\frac{\beta}{m}+\frac{2 n}{r m}+\frac{3 n}{r p}-\frac{1}{p}+\frac{3 n}{r p^{\prime} m}-\frac{1}{p^{\prime} m}+\frac{\gamma}{m}, \quad j=1, \ldots, m .
$$

Theorem 2.1 is proved.

Remark 2.1. It is interesting to note that formally removing all integration by Bergman balls (or sup) in all estimates of all our theorems we arrive at some known assertions on Bergman projection in Bergman type spaces provided earlier in tubular domains (see [19]).

Theorem 2.2. For $1 \leq p<\infty$, the following estimate is valid

$$
\left\|T_{\vec{\alpha}, \beta, \gamma}(g)\right\|_{\left(L_{\overrightarrow{,}, \vec{s}}^{p}\right)_{2}\left(T_{\Omega}^{m}\right)} \leq c\|\mid g\|_{\left(L_{\tau}^{p}\right)\left(T_{\Omega}\right)}^{p},
$$

where $\frac{1}{p}+\frac{1}{p^{\prime}}=1, \beta>-1, p^{\prime} \gamma>-1, s_{j}>\frac{n}{r}-1$,

$$
\alpha_{j}>\frac{\beta}{m}+\frac{2 n}{r m}+\frac{2 n}{r p}-\frac{1}{p}+\frac{3 n}{r p^{\prime} m}-\frac{1}{p^{\prime} m}+\frac{\gamma}{m}+\frac{s_{j}}{p}, \quad j=1, \ldots, m .
$$

and

$$
\tau=p \beta+\frac{2 n m}{r}+\sum_{j=1}^{m}\left(s_{j}-p \alpha_{j}+\nu_{j}\right)+p \gamma+\frac{4 n p}{r p^{\prime}} .
$$

Proof. We need to prove that

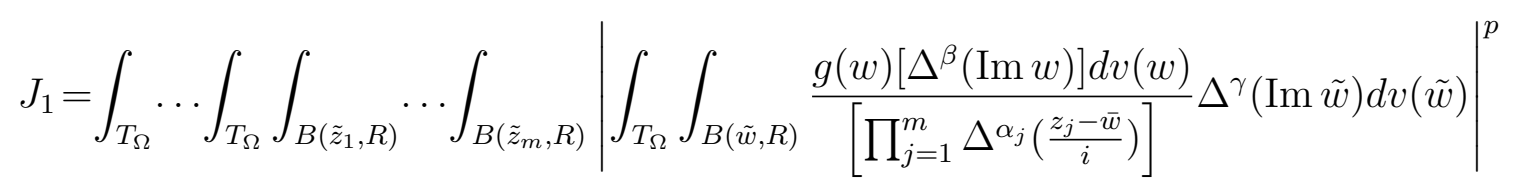




$$
\times \prod_{j=1}^{m} \Delta^{\nu_{j}-\frac{n}{r}}\left(z_{j}\right) d v\left(z_{j}\right) \prod_{j=1}^{m} \Delta^{s_{j}-\frac{n}{r}}\left(\operatorname{Im} \tilde{z}_{j}\right) d v\left(\tilde{z}_{j}\right) \leq c\|\mid g(w)\|_{\left(L_{\tau}^{p}\right)\left(T_{\Omega}\right)} .
$$

Repeating arguments of the proof of Theorem 2.1 we can see that

$$
\left|T_{\vec{\alpha}, \beta, \gamma}(g)\left(z_{1}, \ldots, z_{m}\right)\right|^{p} \prod_{j=1}^{m} \Delta^{\nu_{j}-\frac{n}{r}}\left(z_{j}\right) \leq c_{1} \int_{T_{\Omega}} \int_{B(\tilde{w}, R)} \frac{|g(w)|^{p}\left[\Delta^{\beta}(\operatorname{Im} w)\right] d v(w)}{\left|\prod_{j=1}^{m} \Delta^{p h_{j}}\left(\frac{z_{j}-\bar{w}}{i}\right)\right|} d v(\tilde{w})
$$

with restriction (2.1) on parameters.

In this case using Fubbini's theorem we obtain (see also Theorem 2.1)

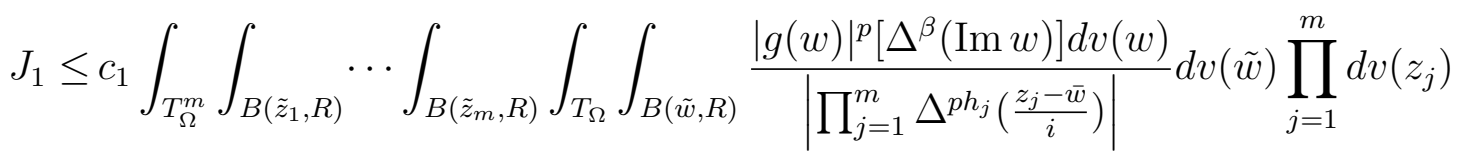

$$
\begin{aligned}
& \times \prod_{j=1}^{m} \Delta^{s_{j}-\frac{n}{r}}\left(\operatorname{Im} \tilde{z}_{j}\right) \leq c_{2} \int_{T_{\Omega}} \int_{B(\tilde{w}, R)}|g(w)|^{p} \Delta^{\beta}(\operatorname{Im} w) \\
& \times \prod_{j=1}^{m} \int_{T_{\Omega}} \int_{B\left(\tilde{z}_{j}, R\right)} \frac{d v\left(z_{j}\right) \Delta^{s_{j}-\frac{n}{r}}\left(\operatorname{Im} \tilde{z}_{j}\right) d v\left(\tilde{z}_{j}\right)}{\left|\Delta^{p h_{j}}\left(\frac{z_{j}-\bar{w}}{i}\right)\right|} d v(w) d v(\tilde{w}) .
\end{aligned}
$$

Now, using (1.2) and Lemma 1.4 we see

$$
\begin{aligned}
J_{1} & \leq c_{2} \int_{T_{\Omega}} \int_{B(\tilde{w}, R)}|g(w)|^{p} \Delta^{\beta}(\operatorname{Im} w) \prod_{j=1}^{m} \int_{T_{\Omega}} \frac{\Delta^{s_{j}-\frac{n}{r}}\left(\operatorname{Im} \tilde{z}_{j}\right) d v\left(\tilde{z}_{j}\right)}{\left|\Delta^{p h_{j}-2 n / r}\left(\frac{z_{j}-\bar{w}}{i}\right)\right|} d v(w) d v(\tilde{w}) \\
& \leq c_{3} \int_{T_{\Omega}} \int_{B(\tilde{w}, R)}|g(w)|^{p} \Delta^{\left(\beta+3 n m / r+\sum_{j=1}^{m}\left(s_{j}-p h_{j}\right)\right.}(\operatorname{Im} w) d v(w) d v(\tilde{w}) \\
& =c_{3} \int_{T_{\Omega}} \int_{B(\tilde{w}, R)}|g(w)|^{p} \Delta^{\tau}(\operatorname{Im} w) d v(w) d v(\tilde{w}),
\end{aligned}
$$

where $\tau=\beta+\sum_{j=1}^{m}\left(s_{j}-p h_{j}\right)+3 n m / r$ and

$$
p h_{j}-s_{j}-\frac{\beta}{m}-\frac{2 n}{r m}-\frac{n}{r}>\frac{n}{r}-1, \quad j=1, \ldots, m .
$$

Using (2.2) and the fact that $h_{j}=\alpha_{j}-b_{j}$ we have

$$
\tau=\beta+\frac{3 n m}{r}+\sum_{j=1}^{m}\left(s_{j}-p \alpha_{j}+\nu_{j}\right)+p \gamma+\frac{p \beta}{p^{\prime}}+\frac{4 n p}{r p^{\prime}}-\frac{n m}{r} .
$$

Moreover from (2.1) and (2.4) we have some restriction on $\alpha_{j}$ :

$$
\alpha_{j}>\frac{\beta}{m}+\frac{2 n}{r m}+\frac{2 n}{r p}-\frac{1}{p}+\frac{3 n}{r p^{\prime} m}-\frac{1}{p^{\prime} m}+\frac{\gamma}{m}+\frac{s_{j}}{p}, \quad j=1, \ldots, m .
$$

Theorem 2.2 is now proved. 
Remark 2.2. In the theorems of this section some new Herz type spaces appeared in tube domains over symmetric cones. We remark similar spaces of analytic functions with quazinorms (norms) $\left\|\sup _{z \in D(\bar{w}, R)}|g(z)|\right\|_{A_{\nu}^{p}(B)}$, where $B$ as usual is the unit ball and $D(w, R)$ is the Bergman ball in Bergman metric in $B$ was considered and studied recently in [9].

Let $\alpha_{j}>0$, let $\beta_{j}>-1$, let $\gamma_{j}>-1, j=1, \ldots, m$. Now we define another new integral Herz type operator

$$
\begin{aligned}
{\left[T_{\vec{\alpha}, \vec{\beta}, \vec{\gamma}}^{1}(g)\right]\left(z_{1}, \ldots, z_{m}\right)=} & \int_{T_{\Omega}} \ldots \int_{T_{\Omega}} \int_{B\left(\tilde{w}_{1}, R\right)} \ldots \int_{B\left(\tilde{w}_{m}, R\right)} g\left(w_{1}, \ldots, w_{m}\right) \\
& \times \frac{\prod_{j=1}^{m}\left[\Delta^{\beta_{j}}\left(\operatorname{Im} w_{j}\right)\right] d v\left(w_{1}\right) \ldots d v\left(w_{m}\right)}{\left[\prod_{j=1}^{m} \Delta^{\alpha_{j}}\left(\frac{z_{j}-\bar{w}_{j}}{i}\right)\right]} \\
& \times \prod_{j=1}^{m} \Delta^{\gamma_{j}}\left(\operatorname{Im} \tilde{w}_{j}\right) d v\left(\tilde{w}_{1}\right) \ldots d v\left(\tilde{w}_{m}\right)
\end{aligned}
$$

where $z_{j} \in T_{\Omega}, j=1, \ldots, m$ for a $g$ function from $L^{1}$ class on product of tubes. These type Bergman integral operators are new even in one dimensional case. In the following theorem we provide another interesting result in this direction which is new even in one dimensional case also.

Theorem 2.3. For $1 \leq p<\infty$, the following estimate is valid

$$
\left\|T_{\vec{\alpha}, \vec{\beta}, \vec{\gamma}}^{1}(g)\right\|_{\left(L_{\vec{\nu}}^{p}\right)_{1}\left(T_{\Omega}^{m}\right)} \leq c\|g\|_{\left(L_{\tau}^{p}\right)\left(T_{\Omega}^{m}\right)}
$$

where $\frac{1}{p}+\frac{1}{p^{\prime}}=1, \beta_{j}>-1, p^{\prime} \gamma_{j}>-1$,

$$
\alpha_{j}>\beta_{j}+\frac{2 n}{r p^{\prime}}+\frac{3 n}{r}+\gamma_{j}-1, \quad j=1, \ldots, m,
$$

and

$$
\tau_{j}=p \beta_{j}+\frac{2 n}{r}-p \alpha_{j}+\nu_{j}+\gamma_{j} p+\frac{4 n p}{r p^{\prime}}+\frac{n}{r}, \quad j=1, \ldots, m .
$$

Proof. We need to prove that

$$
\begin{aligned}
J= & \int_{T_{\Omega}^{m}} \mid \int_{T_{\Omega}^{m}} \int_{B\left(\tilde{w}_{1}, R\right)} \cdots \int_{B\left(\tilde{w}_{m}, R\right)} g\left(w_{1}, \ldots, w_{m}\right) \frac{\prod_{j=1}^{m}\left[\Delta^{\beta_{j}}\left(\operatorname{Im} w_{j}\right)\right] d v\left(w_{1}\right) \ldots d v\left(w_{m}\right)}{\left[\prod_{j=1}^{m} \Delta^{\left.\alpha_{j}\left(\frac{z_{j}-\bar{w}_{j}}{i}\right)\right]}\right.} \\
& \times \prod_{j=1}^{m} \Delta^{\gamma_{j}}\left(\operatorname{Im} \tilde{w}_{j}\right) d v\left(\tilde{w}_{1}\right) \ldots d v\left(\tilde{w}_{m}\right) \mid \prod_{j=1}^{m} \Delta^{\nu_{j}-\frac{n}{r}}\left(z_{j}\right) d v\left(z_{j}\right) \leq c\|g\|_{\left(L_{\tau}^{p}\right)\left(T_{\Omega}\right)} .
\end{aligned}
$$

Let us denote

$$
\int_{B\left(\tilde{w}_{1}, R\right)} \cdots \int_{B\left(\tilde{w}_{m}, R\right)}=\int_{B^{m}(W, R)}
$$


and

$$
g\left(w_{1}, \ldots, w_{m}\right)=g(\vec{w}) .
$$

Using Holder's inequality we have

$$
\begin{aligned}
\left|T_{\vec{\alpha}, \vec{\beta}, \vec{\gamma}}^{1}(g)\left(z_{1}, \ldots, z_{m}\right)\right|= & \int_{T_{\Omega}^{m}}\left(\int_{B^{m}(W, R)} \frac{|g(\vec{w})|^{p}\left[\prod_{j=1}^{m} \Delta^{\beta_{j}}\left(\operatorname{Im} w_{j}\right)\right] d v\left(w_{1}\right) \ldots d v\left(w_{m}\right)}{\left|\prod_{j=1}^{m} \Delta^{p h_{j}}\left(\frac{z_{j}-\bar{w}_{j}}{i}\right)\right|}\right)^{\frac{1}{p}} \\
& \times\left(\int_{B^{m}(W, R)} \frac{\left[\prod_{j=1}^{m} \Delta^{\beta_{j}}\left(\operatorname{Im} w_{j}\right)\right] d v\left(w_{1}\right) . . d v\left(w_{m}\right)}{\left|\prod_{j=1}^{m} \Delta^{p^{\prime} b_{j}}\left(\frac{z_{j}-\bar{w}_{j}}{i}\right)\right|}\right)^{\frac{1}{p^{\prime}}} \\
& \times \prod_{j=1}^{m} \Delta^{\gamma_{j}}\left(\operatorname{Im} \tilde{w}_{j}\right) d v\left(\tilde{w}_{1}\right) \ldots d v\left(\tilde{w}_{m}\right),
\end{aligned}
$$

where $h_{j}+b_{j}=\alpha_{j}, \frac{1}{p}+\frac{1}{p^{\prime}}=1, j=1, \ldots, m$.

Such as integration variables divided, by Lemma 1.4

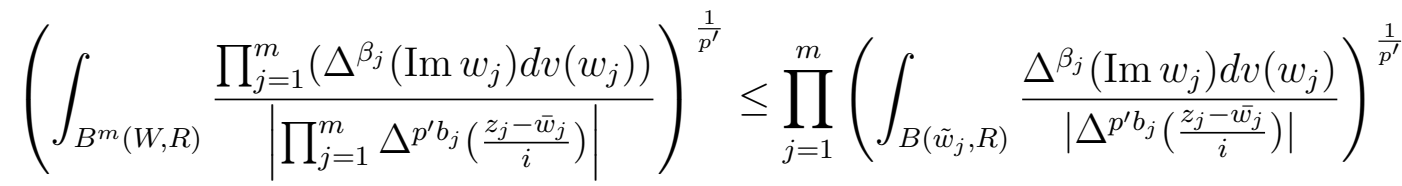

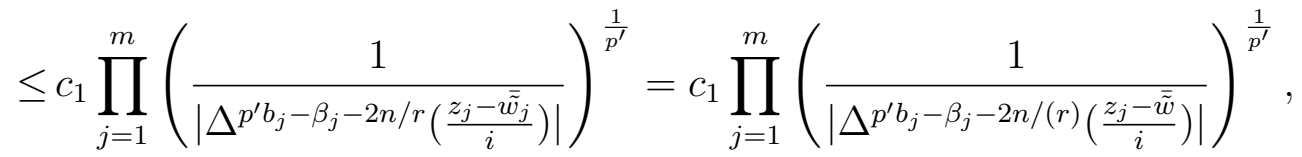

where $\beta_{j}>-1$ and $p^{\prime} b_{j}>\beta_{j}+\frac{2 n}{r}$. So we have

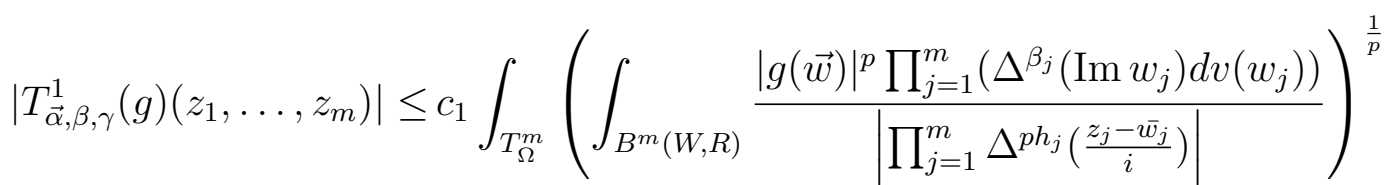

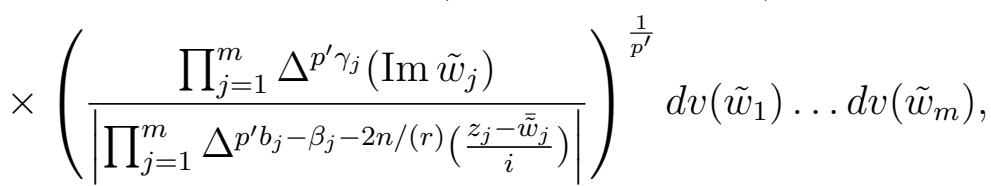

$z_{j} \in T_{\Omega}, j=1, \ldots, m$.

Let us use Holder inequality once more

$$
\begin{aligned}
\left|T_{\vec{\alpha}, \beta, \gamma}^{1}(g)\left(z_{1}, \ldots, z_{m}\right)\right|= & \left(\int_{T_{\Omega}^{m}} \int_{B^{m}(W, R)} \frac{|g(\vec{w})|^{p} \prod_{j=1}^{m}\left(\Delta^{\beta_{j}}\left(\operatorname{Im} w_{j}\right) d v\left(w_{j}\right)\right)}{\left|\prod_{j=1}^{m} \Delta^{p h_{j}}\left(\frac{z_{j}-\bar{w}_{j}}{i}\right)\right|_{j=1}^{m}} \prod_{j} d v\left(\tilde{w}_{j}\right)\right)^{\frac{1}{p}} \\
& \times\left(\int_{T_{\Omega}^{m}} \frac{\prod_{j=1}^{m}\left(\Delta^{p^{\prime} \gamma_{j}}\left(\operatorname{Im} \tilde{w}_{j}\right) d v\left(\tilde{w}_{j}\right)\right)}{\left.\mid \prod_{j=1}^{m} \Delta^{p^{\prime} b_{j}-\beta_{j}-2 n /(r)\left(\frac{z_{j}-\overline{\tilde{w}}_{j}}{i}\right) \mid}\right)^{\frac{1}{p^{\prime}}} .}\right.
\end{aligned}
$$


And so

$$
\begin{aligned}
\left|T_{\vec{\alpha}, \beta, \gamma}^{1}(g)\left(z_{1}, \ldots, z_{m}\right)\right|^{p} \leq & c_{1} \int_{T_{\Omega}^{m}} \int_{B^{m}(W, R)} \frac{|g(\vec{w})|^{p} \prod_{j=1}^{m}\left(\Delta^{\beta_{j}}\left(\operatorname{Im} w_{j}\right) d v\left(w_{j}\right)\right)}{\left|\prod_{j=1}^{m} \Delta^{p h_{j}}\left(\frac{z_{j}-\bar{w}_{j}}{i}\right)\right|} \prod_{j=1}^{m} d v\left(\tilde{w}_{j}\right) \\
& \times\left(\prod_{j=1}^{m} \int_{T_{\Omega}} \frac{\left(\Delta^{p^{\prime} \gamma_{j}}\left(\operatorname{Im} \tilde{w}_{j}\right) d v\left(\tilde{w}_{j}\right)\right)}{\left.\mid \Delta^{p^{\prime} b_{j}-\beta_{j}-2 n /(r)\left(\frac{z_{j}-\tilde{\tilde{w}}_{j}}{i}\right) \mid}\right)^{\frac{p}{p^{\prime}}}} \cdot\right.
\end{aligned}
$$

But with respect to (1.2) we have

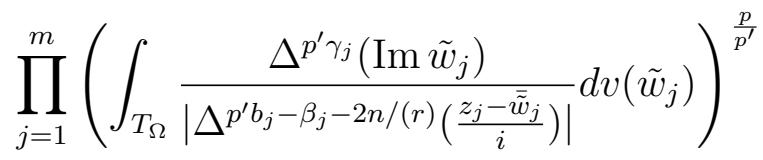

$$
\begin{aligned}
& \leq c_{2} \prod_{j=1}^{m} \Delta^{\left(p^{\prime} \gamma_{j}-p^{\prime} b_{j}+\beta_{j}+2 n / r+2 n /(r m)\right) p /\left(p^{\prime}\right)}\left(\operatorname{Im} z_{j}\right)=c_{2} \prod_{j=1}^{m} \Delta^{p \gamma_{j}-p b_{j}+\beta_{j} p / p^{\prime}+4 n p /\left(r p^{\prime}\right)}\left(\operatorname{Im} z_{j}\right) \text {. }
\end{aligned}
$$

Where $p^{\prime} \gamma_{j}>-1$ for $j=1, \ldots, m$ and

$$
p^{\prime} b_{j}-p^{\prime} \gamma_{j}-\beta_{j}-\frac{4 n}{r}>\frac{n}{r}-1
$$

Denote now $p \gamma_{j}-p b_{j}+\beta_{j} p / p^{\prime}+4 n p /\left(r p^{\prime}\right)=-\nu_{j}+\frac{n}{r}, j=1, \ldots, m$. Then

$$
b_{j}=\frac{\nu_{j}}{p}+\gamma_{j}+\frac{\beta_{j}}{p^{\prime}}+\frac{2 n}{r m p^{\prime}}+\frac{2 n}{r p^{\prime}}-\frac{n}{r p} .
$$

In this case we have

$$
J \leq c_{3} \int_{T_{\Omega}^{m}} \int_{T_{\Omega}^{m}} \int_{B^{m}(W, R)} \frac{|g(\vec{w})|^{p} \prod_{j=1}^{m}\left(\Delta^{\beta_{j}}\left(\operatorname{Im} w_{j}\right) d v\left(w_{j}\right)\right)}{\left|\prod_{j=1}^{m} \Delta^{p h_{j}}\left(\frac{z_{j}-\bar{w}}{i}\right)\right|} \prod_{j=1}^{m} d v\left(\tilde{w}_{j}\right) \prod_{j=1}^{m} d v\left(z_{j}\right) .
$$

Using Fubbini's theorem we obtain

$$
\begin{aligned}
& J \leq c_{3} \int_{T_{\Omega}^{m}} \int_{B^{m}(W, R)}|g(\vec{w})|^{p} \prod_{j=1}^{m}\left(\int_{T_{\Omega}} \frac{d v\left(z_{j}\right)}{\mid \Delta^{p h_{j}\left(\frac{z_{j}-\bar{w}_{j}}{i}\right) \mid}}\left(\Delta^{\beta_{j}}\left(\operatorname{Im} w_{j}\right)\right) \prod_{j=1}^{m} d v\left(w_{j}\right)\right. \\
& \times \prod_{j=1}^{m} d v\left(\tilde{w}_{j}\right) \leq c_{4} \int_{T_{\Omega}^{m}} \int_{B^{m}(W, R) \mid}|g(\vec{w})|^{p} \prod_{j=1}^{m}\left(\Delta^{\beta_{j}+2 n / r-p h_{j}}\left(\operatorname{Im} w_{j}\right) d v\left(w_{j}\right)\right) d v(\tilde{w}) \\
& =c_{4} \int_{T_{\Omega}^{m}} \int_{B^{m}(W, R) \mid}|g(\vec{w})|^{p} \prod_{j=1}^{m}\left(\Delta^{\tau_{j}}\left(\operatorname{Im} w_{j}\right) d v\left(w_{j}\right)\right) \prod_{j=1}^{m} d v\left(\tilde{w}_{j}\right),
\end{aligned}
$$

where $\tau_{j}=\beta_{j}-p h_{j}+2 n / r, j=1, \ldots, m$ and

$$
p h_{j}-\beta_{j}-\frac{2 n}{r}>\frac{n}{r}-1 .
$$

Using (2.6) and fact that $h_{j}=\alpha_{j}-b_{j}$ we have

$$
\tau_{j}=\beta_{j}+\frac{2 n}{r}-p \alpha_{j}+\nu_{j}+\gamma_{j} p+\frac{p \beta}{p^{\prime}}+\frac{4 n p}{r p^{\prime}}+\frac{n}{r}, \quad j=1, \ldots, m .
$$


Moreover from (2.5) and (2.7) we have some restriction on $\alpha_{j}$ :

$$
\alpha_{j}>\beta_{j}+\frac{2 n}{r p^{\prime}}+\frac{3 n}{r}+\gamma_{j}-1, \quad j=1, \ldots, m .
$$

Theorem 2.3 is proved.

Similarly we may prove the following result.

Theorem 2.4. For $1 \leq p<\infty$, the following estimate is valid

$$
\left\|T_{\vec{\alpha}, \vec{\beta}, \vec{\gamma}}^{1}(g)\right\|_{\left(L_{\vec{\nu}}^{p}\right)_{2}\left(T_{\Omega}^{m}\right)} \leq c\|g\|_{\left(L_{\tau}^{p}\right)\left(T_{\Omega}^{m}\right)},
$$

where $\frac{1}{p}+\frac{1}{p^{\prime}}=1, \beta_{j}>-1, p^{\prime} \gamma_{j}>-1$,

$$
\alpha_{j}>\beta_{j}+\gamma_{j}+\frac{s_{j}}{p}-1+\frac{3 n}{r}+\frac{n}{p r}+\frac{2 n}{r p^{\prime}}, \quad j=1, \ldots, m,
$$

and

$$
\tau_{j}=p \beta_{j}+\frac{2 n p}{r}+s_{j}-p \alpha_{j}+\nu_{j}+p \gamma_{j}+\frac{2 n p}{r p^{\prime}}, \quad j=1, \ldots, m .
$$

In our following work we hope to turn more closely to issues related with the sharpness of these interesting results.

Acknowledgements. This work was supported by the Ministry of Education and Science of the Russian Federation (grant 1.1704.2014K).

\section{REFERENCES}

[1] M. Abate, J. Raissy and A. Saracco, Toeplitz operators and Carleson measures in strongly pseudoconvex domains, J. Funct. Anal. 263 (2012), 3449-3491.

[2] M. Andersson and H. Carlsson, $Q_{p}$ spaces in strictly pseudoconvex domains, J. Anal. Math. 84 (2001), 335-359

[3] F. Beatrous, $L^{p}$ estimates for extensions of analytic functions, Michigan Math. J. 32 (1985), 134-161.

[4] D. Békollé,, A. Bonami, G. Garrigós, F. Ricci and B. Sehba, Analytic Besov spaces and Hardy type inequalities in tube domains over symmetric cones J. Reine Angew. Math. 647 (2010), $25-56$.

[5] D. Békollé, A. Bonami, G. Garrigós, C. Nana, M. Peloso and F. Ricci, Lecture notes on Bergman projectors in tube domain over cones, an analytic and geometric viewpoint, IMHOTEP J. Afr. Math. Pures Appl. 5, 2004.

[6] D. Békollé, A. Bonami, G. Garrigós and F. Ricci, Littlewood-Paley decomposition and Besov spaces related to symmetric cones and Bergman Projections in Tube Domains, Proc. Lond. Math. Soc. 89(2) (2004), 317-360.

[7] D. Békollé and A. Kagou, Molecular decomposition and interpolation, Integral Equations Operator Theory 31 (1998), 150-177.

[8] D. Békollé and A. Kagou, Reproducing properties and $L^{p}$-estimates for Bergman projections in Siegel domains of type II, Studia Math. 115(3) (1995), 219-239.

[9] Z. Chen and W. Quyang, Maximal and area characterizations of Bergman spaces in the unit ball, J. Funct. Spaces 2013 (2013), 1-13. 
[10] D. Debertol, Besov spaces and boundedness of weighted Bergman projections over symmetric tube domains, PhD Thesis, University of Genova, 2003.

[11] M. M. Djrbashian and F. A. Shamoian, Topics in the theory of $A_{\alpha}^{p}$ classes, Teubner Texte zur Mathematics 105 (1988).

[12] M. Englis, T. Hannimen and T. Taskinen, Minimal $L^{\infty}$ type on strictly pseudoconvex domains on which Bergman projection continious, Houston J. Math, 32 (2006), 253-275.

[13] J. Faraut and A. Koranyi, Analysis on symmetric cones, Oxford Mathematical Monographs, The Clarendon Press, New York, 1994.

[14] M. Jevtic, R. F. Shamoyan and M. Pavlovic, A note on the diagonal mapping in spaces of analytic functions in the unit polydisc, Publ. Math. Debrecen 74 (2009), 45-58.

[15] C. Nana and B. Sehba, Carleson embeddings and two operators on Bergman spaces of tube domains over symmetric cones, Integral Equations Operator Theory 83 (2015), 151-178.

[16] J. Ortega and J. Fabrega, Hardy's inequality and embeddings in holomorphic Triebel-Lizorkin spaces, Illinois J. Math. 43 (1999), 733-751.

[17] J. Ortega and J. Fabrega, Mixed norm spaces and interpolation, Studia Math. 109(1994), 233254

[18] J. Ortega and J. Fabrega, Pointwise multipliers and corona type decomposition in BMOA, Ann. Inst. Fourier (Grenoble) 46 (1996), 111-137.

[19] B. F. Sehba, Bergman type operators in tube domains over symmetric cones, Proc. Edinburg. Math. Society, 52 (2009), 529-544.

[20] B. F. Sehba, Hankel operators on Bergman spaces of tube domains over symmetric cones, Integral Equations Operator Theory 62 (2008), 233-245.

[21] R. Shamoyan and O. Mihić In search of traces of some holomorphic functions in polyballs, Revista Notas de Matemática 4 (2008), 1-23.

[22] R. Shamoyan and O. Mihić On traces of holomorphic functions on the unit polyball, Appl. Anal. Discrete Math. 3 (2009), 198-211.

[23] R. Shamoyan and S. Kurilenko, On traces of Herz-type and Bloch-type spaces in bounded pseudoconvex domains, Probl. Anal. Issues Anal. 4 (2015), 73-94.

[24] S. Yamaji, Positive Toeplitz operators on the Bergman spaces in minimal bounded homogeneous domians, Hokkaido Math. J. 41 (2012), 257-274.

${ }^{1}$ Brjansk State University

BEJICKAYA, 79

BRYANSK, 241036

RUSSIA

E-mail address: SergKurilenko@gmail.com, rsham@mail.ru 